\title{
8-2011
}

\section{Novel Irradiated Axial Rotational Flap Model in the Rodent}

\author{
Adam J. Luginbuhl \\ Thomas Jefferson University \\ Mara Modest, BS \\ Thomas Jefferson University \\ Kaiguo Yan \\ Thomas Jefferson University \\ Joseph Curry \\ Thomas Jefferson University \\ Ryan N. Heffelfinger \\ Thomas Jefferson University \\ Follow this and additional works at: https://jdc.jefferson.edu/otofp \\ Part of the Otolaryngology Commons \\ Let us know how access to this document benefits you
}

\section{Recommended Citation}

Luginbuhl, Adam J.; Modest, BS, Mara; Yan, Kaiguo; Curry, Joseph; and Heffelfinger, Ryan N., "Novel Irradiated Axial Rotational Flap Model in the Rodent" (2011). Department of

Otolaryngology - Head and Neck Surgery Faculty Papers. Paper 21.

https://jdc.jefferson.edu/otofp/21

This Article is brought to you for free and open access by the Jefferson Digital Commons. The Jefferson Digital Commons is a service of Thomas Jefferson University's Center for Teaching and Learning (CTL). The Commons is a showcase for Jefferson books and journals, peer-reviewed scholarly publications, unique historical collections from the University archives, and teaching tools. The Jefferson Digital Commons allows researchers and interested readers anywhere in the world to learn about and keep up to date with Jefferson scholarship. This article has been accepted for inclusion in Department of Otolaryngology - Head and Neck Surgery Faculty Papers by an authorized administrator of the Jefferson Digital Commons. For more information, please contact: JeffersonDigitalCommons@jefferson.edu. 


\title{
As submitted to:
}

Otolaryngology-Head and Neck Surgery

And later published as:

\section{Novel Irradiated Axial Rotational Flap Model in the Rodent}

\author{
Volume 145, Issue 2, suppl P49, August 2011 \\ doi: 10.1177/0194599811416318a27 \\ Adam Luginbuhl MD ${ }^{1}$, Mara Modest BS ${ }^{1}$, Kaiguo Yan PhD², Joseph Curry MD ${ }^{1}$ Ryan \\ Heffelfinger $\mathrm{MD}^{1}$
}

1Thomas Jefferson University, Dept of Otolaryngology Head and Neck Surgery, Philadelphia PA

2Thomas Jefferson University, Dept of Radiation Oncology, Philadelphia PA

Conflict of Interest: None

Disclosure: None

\section{Corresponding Author:}

Adam Luginbuhl, MD

Thomas Jefferson University Hospital

Otolaryngology / Head and Neck Surgery

925 Chestnut St. 6th Floor

Philadelphia, PA 19107

Phone: 215-240-0748

Fax: 215-923-4532

aluginbuhl@gmail.com 
Oral Presentation at American Academy of Otolaryngology Annual meeting September 11, 2011, San Francisco, CA

Word Count : 2,228 
Abstract

Objectives: To design an easily reproducible rodent rotational skin flap and to evaluate the effects of radiation on flap viability.

Methods: Ten rats received 40 Gy irradiation to the abdominal wall. Following a recovery period of one month, a 3 X $8 \mathrm{~cm}$ fasciocutaneous flap based axially on the inferior epigastric vessel, was raised and rotated 60 degrees into a contralateral deficit. Five non-irradiated rats underwent the identical procedure as a control. Animals were sacrificed seven days postoperatively, areas of flap necrosis were documented, and histological specimens were taken to compare flap viability and vessel density.

Results: $60 \%$ of the rats in the irradiated group had necrosis of the distal flap ranging from 1 to $6 \mathrm{~cm}$ from the distal edge, whereas none of the animals (0\%) in the control group exhibited necrosis $(\mathrm{p}<0.001)$. Histology revealed collagen and vascular changes in the irradiated skin. Vascular density analysis revealed a significant difference between radiated and non-radiated flaps; $\mathrm{p}=0.004,0.029$ and 0.014 in the distal, middle and proximal segments of the flap respectively.

Conclusion: This novel rat axial rotational flap model demonstrates increases flap necrosis and a decrease in vascular density due to the effects of radiation. Using a linear electron accelerator a dose of 40 gy can be delivered to the skin without resulting in devastating gastrointestinal side effects. 


\section{Introduction}

An increasing proportion of patients who require extensive surgical resection of head and neck malignancies have a history of prior treatment with radiation and chemotherapy. Chemoradiation-induced damage to the skin and soft tissues includes increased fibrosis and a diminished capillary blood supply that dramatically increases the rate of fistula formation, skin breakdown, and wound dehiscence following surgical reconstruction. Local rotational flaps can develop partial distal necrosis in the immediate postoperative period with as high as 31\% partial flap loss in pedicled musculocutaneous flaps used for head and neck, as well as breast reconstruction'1. Management of these complications generally requires either further surgery or prolonged wound care, which can significantly delay further cancer treatment and increases the overall cost of treatment.

In order to develop new therapeutic options to prevent flap failure, the understanding of flap physiology is imperative. Three perfusion zones contribute to healthy skin flap physiology and ultimate viability. Zone I is the pedicle to the flap, zone II is at the capillary level, and zone III is the interstitial space where the process of nutritional delivery takes place. In zones II and III of normal healthy tissue, the interstitial space is filled with proteoglycans and collagen with evenly dispersed capillary network, unlike with irradiated tissue, where this space is replaced by fibrosis and the relative water content and capillary content is greatly diminished ${ }^{2,3}$.

The published literature describes several different rat flaps ranging from random to free tissue models. In all of these studies, flaps are designed to demonstrate maximum necrosis in the control group with endpoints of improved flap survival over the control group. Multiple studies demonstrate necrosis in the control group ${ }^{4-8}$. This stands in 
opposition to the primary goal of clinical flap design, which is to ensure $100 \%$ flap survival without necrosis. We felt it important that our model correspond to clinical practice, so in the current study, the non-irradiated rats (control group) had a 100\% flap survival from which we could test our hypothesis that radiation would result in development of flap compromise and necrosis.

The primary objective of this study was to create a local rotational flap in the irradiated rodent model mimicking the rotational flaps used in head and neck reconstruction. Secondary objectives included evaluating if irradiation of this model resulted in flap failure and necrosis and to characterize any changes in vascular density.

\section{Methods:}

\section{Animals}

Fifteen male Sprague-Dawley rats, weighing 600+/- 200 grams were used. The protocol was approved by the Thomas Jefferson University Institutional Animal Care and Use Committee. Animals were housed with a 12-h light/dark cycle with food and water ad libitum. Animals were monitored daily following any irradiation or surgical procedure.

\section{Radiation Protocol}

Ten rats received 40 Gy electron beam irradiation to their ventral abdominal wall while 5 rats received no radiation and served as a control. The ventral abdomen of all rats (including those not irradiated) was shaved while under isofluorane inhalation anesthesia. After 7 days of acclimation, rats receiving radiation were anesthesized via subcutaneous 
injection of $80 \mathrm{mg} / \mathrm{ml}$ of ketamine, $1.6 \mathrm{mg} / \mathrm{ml}$ of acepromazine, and $5 \mathrm{mg} / \mathrm{ml}$ of Xylazine, with supplementation as needed. Once anesthesia was confirmed, four rats at a time were placed in the supine position on the irradiation table(figure 1).

A 6-MeV electron beam accelerator (Electra) was used to radiate the dermis and epidermis. For the animals receiving 40 Gy, irradiation was administered over 8 days in four doses of 10 Gy given every other day. In order to deliver the intended dose to the dermis without delivering toxic radiation to the alimentary tract, the steep part of the dosimetry curve was used with a rapid fall to $2 \mathrm{~Gy}$ at $2 \mathrm{~cm}$ below the epidermis (figure 1a). This is achieved by delivering 20 Gy using $2.59 \mathrm{~cm}$ of $\mathrm{H} 20$ equivalent shielding to achieve a $50 \%$ dose (10 Gy) at the skin and $10 \%$ dose ( $2 \mathrm{~Gy}$ ) at the gut. A $25 \times 25 \mathrm{~cm}$ cone was used with the torso and head outside of the beam and the genitals and lower limbs being shielded by the equivalent of $1 \mathrm{~cm}$ of $\mathrm{H} 20$ (Figure $1 \mathrm{~b}$ ).

Rats were given saline injections for rehydration following each dose of radiation and their weights monitored for 28 days following the last irradiation treatment.

\section{Ventral Fasciocutaneous Rotational Flap Procedure}

Rats were allowed to recover from the irradiation treatment for one month prior to undergoing the ventral fasciocutaneous rotational flap procedure. Isofluorane inhalation was used for all rats during the procedure and $0.25 \mathrm{cc}$ dose of Buprenorphine was administered prior to the first incision. Initially, the approximate location of the 
inferior epigastric artery was marked. A 3 X $8 \mathrm{~cm}$ flap was drawn with the medial part of the flap drawn down the midline of the abdomen $8 \mathrm{~cm}$ from the origin of the inferior epigastric. The lateral extent of the flap was $3 \mathrm{~cm}$ from midline.

Using sterile technique, incisions were carried out along the medial, superior, and lateral sides, leaving the inferior part of the flap attached with the pedicle. The plane of dissection was superficial to the rectus abdominus fascia. All bridging vessels, as well as the superior epigastric vascular pedicle, were ligated. Dissection was carried out in this avascular plane until the inferior epigastric pedicle was visualized(figure 2).

A defect was created on the left half of the abdomen. From the point of the right inferior epigastric pedicle an incision 60 degrees off the midline was carried for $8 \mathrm{~cm}$ to create a 3 X $8 \mathrm{~cm}$ defect. The flap was then rotated into this defect, sutured into place, and the donor defect was closed primarily using 4.0 vicryl buried mattress sutures followed by a running suture along the entire perimeter (Figure 2). Buprenorphine was administered for postoperative analgesia every 12 hours for two days.

We determined the appropriate flap and defect size by varying flap and defect dimensions in non-irradiated rats until necrosis occurred. Using a separate cohort of rats, the above procedure was performed with flaps $3 \mathrm{~cm}$ wide by 7,8 , and $9 \mathrm{~cm}$ long. The defects were also varied accordingly. Necrosis developed when the flaps and defects were greater than $8 \mathrm{~cm}$. Based on these results we established that the limits of this axial flap were a 3 X $8 \mathrm{~cm}$ flap rotated into a 3 X $8 \mathrm{~cm}$ defect. We then proceeded to test whether radiation would lead to flap necrosis. Animals were monitored for 7 days postoperative. Prior to sacrifice, pictures were taken and the flaps were harvested for future histological examination. 


\section{Evaluation of Flap Revascularization}

Seven days following flap elevation, Animals were reanesthetized as previously described and flap viability was evaluated using planimetry. Each flap was harvested and a standardized digital photograph was taken of each ventral flap (NikonD60). These were then digitally processed and a qualified blinded observer measured the amount of necrosis from the distal tip of the flap to the junction of viable tissue. Necrotic non-viable areas were defined as dry, hairless eschar, while healthy viable areas were defined as viable pink tissue.

\section{Microvascular Density}

Once photographed, the distal, middle and proximal one $\mathrm{cm}$ sections of the flaps were harvested and then fixed with formalin and embedded in paraffin. These sections were then processed via immunohistochemistry for von Willebrand factor (vWF). Vascularity was assessed by counting blood vessels marked with vWF in eight high-power fields per tissue section and then analyzed using a wilcoxon rank sum test. Animals were excluded from the study if they developed a hematoma or self mutilated their flap. X-ray paper vests were fashioned to prevent the rat from picking at the suture line ${ }^{9}$.

\section{Results:}

There was a significant difference in the survival of skin flaps between the rats that did not receive irradiation compared to those who did (40Gy in four fractions of 10Gy). Sixty percent of the rats in the irradiated group had necrosis of the distal flap ranging from 1-6 $\mathrm{cm}$ from the distal edge compared to $0 \%$ in the controls where no necrosis was seen $(\mathrm{p}<0.001)($ figure 3). Radiated tissue outside of the necrotic distal tip consistently presented with loss of nuclear detail, inflammatory infiltrates, thickened vascular walls, 
atrophy of skeletal muscle, and interstitial fibrosis. Histological analysis of the necrotic tissue in the irradiated animals demonstrated loss of epidermis, necrosis, and vessel thrombosis (Figure 4). In the control group, inflammatory infiltrates were observed in the distal tip region, but without any evidence of necrosis.

Vascular density was significantly affected by radiation. In the control group, the proximal, middle, and distal segments of the flap had a mean value of 185, 209, and 162 vessels/8 high power fields respectively. Whereas the radiated rats had a mean value of only 86,85 , and 79 vessels/8 high power fields (figure 4). Wilcoxon testing of the proximal, middle, and distal segment vascular density resulted in p values of $0.004,0.029$ and 0.014 respectively(figure 5).

\section{Discussion}

The objective of this current study was to use the rodent model to simulate the effects of radiation on normal tissue when stressed by surgical intervention. There is no current proactive or preventative solution to the well-established clinical problem of dramatic increases in wound complications seen in irradiated patients. Conceptually, the ability to revitalize irradiated tissue prior to surgery would decrease the rate of complications post-operatively. Regardless of the complexity of reconstruction, whether primary closure or free tissue transfer, the quality of the local host tissue is integral to the success of the operation. The model in the current study was developed based on the concepts and principles of the cervical facial rotational or advancement flap where tissue surrounding the resection site is undermined and rotated to close the defect. Developing models that mimic clinical experience is one of the initial steps in testing hypotheses that may alter this challenging clinical course. 
With radiation dosing for primary cancer sites and nodal basins reaching 60-70 Gy, dosimetry predicts a dose of 40-50 Gy to the skin. During treatment, patients can experience de-epithelization with occasional ulceration of the skin. Once the acute phase of healing has occurred post radiation, the skin remodels with dense fibrosis, reduced peptidoglycans, and a loss of skin turgor, all leading to the classic "woody neck" 2 . The abdominal skin in the radiated animals of the present study developed moderate epidermolysis with occasional ulceration at two weeks post radiation. These areas were fully healed by 30 days post radiation, which was the time of the flap creation. Previous radiation studies in the rodent have used 30 days post radiation as the point of flap creation due to the increased metabolic rate of the rat ${ }^{6}$. Angelos et al irradiated rats with either 23 Gy or 40 Gy and found a significant delay in free flap revascularization with 40 Gy but not with 23 Gy, thus establishing that 40 Gy effectively demonstrates poor wound healing in the free myocutaneous gracilis flap model ${ }^{6}$. Nall et al was unable to find a deleterious effect of wound healing on animals irradiated with 15 or $25 \mathrm{~Gy}^{7}$.

Fibroblasts in healthy tissue are pivotal in reacting to tissue stressors and optimizing survival. At a fundamental level, the absolute loss of fibroblasts seen with radiation, and their replacement with fibrosis, thwarts this survival process and the demands of wound healing cannot be met. With the aid of a skin chamber model, Hori et al. induced neovascularization in rodent skin using polyvinyl chloride. They subsequently irradiated the area with 10 Gy and discovered a dramatic decrease in the density of capillaries over 96 hours as well as a narrowing of normal vessel caliber ${ }^{10}$. This and other studies demonstrate the diminishing effect of radiation on existing blood vessels. Our model is consistent with Hori et al's findings, in that a dramatic decrease in overall vascular density was observed in the irradiated group ${ }^{10}$. The difference was seen throughout the length of the flap from the proximal, middle, and distal locations. Within 
the non-irradiated group, maximum vessel density was found within the middle portion of the flap, which can be explained by the relative ischemic environment induced by the creation of the flap leading to a pro-angiogenic response (figure 5).

Limitations of our study include the timing of surgery after radiation and small number of animals. Clinically, patients do not undergo surgical interventions 1 month post radiation treatment, yet due to the elevated metabolism of the rat, complete resolution of all acute changes and histologic evidence of fibrosis we feel this model provides insight into the remodeling that takes place with radiation. These changes ultimately affect the outcomes of surgical interventions and this easily reproducible model will allow for further investigation into novel therapies to improve outcomes.

With the development of the axial rotational flap in the irradiated rodent model in the current study, future efforts are aimed at increasing both capillary delivery and diffusion capacity through the interstitial space via a variety of therapeutic interventions. Unlike Schulltze-Mosgau and others, who have looked at modifying tissue behavior at the time of radiation, our focus will be well after radiation has taken place: 7-10 days prior to a planned surgical intervention. The irradiated rat model of an axial rotational flap is consistent with what is seen in previously irradiated patients that develop skin necrosis after reconstruction with local rotational flaps(figure 3). Based on these findings, efforts are now focused on the manipulation of growth factors, heat shock proteins, and the exploitation of adipose stem cells to revitalize irradiated tissue in this novel flap model. 


\section{References:}

1) Zhang F, Fischer K, Komorwska E, Guo M, Lineaweaver W. et al. Improvement of skin paddle survival by application of vascular endothelial growth factor in a rat TRAM flap model. Ann Plastic Surgery 2001; 46: 314-319.

2) Stone $\mathrm{H}$, Coleman $\mathrm{N}$, Anscher $\mathrm{M}$, McBride. Effects of radiation on normal tissue: consequences and mechanisms. Lancet Oncology 2003; 4: 529-536.

3) Schultze-Mosgau s, grabenbauer GG et al Histomorphological structural changes of head and neck blood vessels after pre-or postoperative radiotherapy. Strahlenther Onkol 2002; 178: 299-306

4) Davis, R, Wachholz, J, Jassir D, et al. Comparison of topical anti-ischemic agents in the salvage of failing random-pattern skin flaps in rats. Arch facial Plast Surg 1; 1999: 27-32.

5) Kubota, J. Effects of diode laser therapy on blood flow in axial pattern flaps in the rat model. Lasers Med Science 17; 2002: 146-153.

6) Angelos P, McCarn K, Winn S. Development of an irradiated rodent model to study flap revascularization. Arch of facial Plast Surgery 12 2010; 119-122.

7) Nall A. Brownlee R. Colvin P, et al. Transforming growth factor Beta1 improves wound healing and random flap survival in normal and irradiated rats. Arch of Otolaryngology Head Neck Surgery 122: 1996: 171-177.

8) Harder Y, Amon M, Schramm R, et al. Ischemia-induced up-regulation of heme oxygenase-1 protects from apoptotic cell death and tissue necrosis. Journal of Surgical Research 150; 2008: 293-303.

9) Bayramicli M, Yitmaz B, Numanoglu A. Modified protective rat vest. Plastic and Reconstructive Surgery 1998; 101:859. 
10) Hori, K, Saito, S, Tamai, M. Effect of irradiation on neovascularization in rat skinfold chambers: Implications for clinical trials of low dose radiotherapy for wet-type agerelated macular degeneration. Int. J. radiation Oncology Biol. Phys. 60;2004: 1564-1571. 


\section{Figure Legend:}

Figure 1: a) Dosimetry Curve - Dot indicates the necessary shielding thickness where 50\% of the dose is delivered on the Elekta linear acelerator to deliver the appropreate dose at the skin and then rapidly fall off to limit irradiation to the gut. b) Holding chamber.

Figure 2: a) $8 \times 3 \mathrm{~cm}$ flap and defect, Angle a = 60 degrees. b) Identified inferior epigastric pedicle.

Figure 3: a) Radiated flap at POD\#7 and b) non-radiated flap at POD\#7.

Figure 4: Immunohistochemistry staining for von Willabrand factor vessels outlined by dark staining a) non-radiated, b) radiated. Note dense fibrosis. C) Radiated tissue necrosis of epidermal elements, no definable viable cells. D) Fibrosis of proximal radiated tissue. Muscular layer replaced by fibrosis.

Figure 5: Vascular density of proximal, middle and distal segments. \# vessels countered per 8 high power fields. ${ }^{*} \mathrm{p}=0.004,{ }^{* *} \mathrm{p}=0.029$ and ${ }^{* * *} \mathrm{p}=0.014$. 